\title{
Erratum to: Liquid Biopsy in Cancer Patients: The Hand Lens for Tumor Evolution
}

Antonio Russo, Antonio Giordano, and Christian Rolfo

\section{Erratum to:}

A. Russo et al. (eds.), Liquid Biopsy in Cancer Patients, Current Clinical Pathology, DOI 10.1007/978-3-319-55661-1

The order of the volume editors in the published book was incorrect in the frontmatter and cover. The order has been revised to reflect the wishes of the editors. 